\title{
SELETIVIDADE DE TRIFLURALIN INCORPORADO AO SOLO PARA BATATA-DOCE $\left({ }^{1}\right)$
}

\author{
DOMINGOS ANTONIO MONTEIRO $\left({ }^{2}\right)$, VALDEMIR ANTONIO PERESSIM $\left({ }^{2,4}\right)$, \\ JOSÉ OSMAR LORENZI ( $\left.{ }^{2}\right)$ e DILERMANDO PERECIN $\left({ }^{3,5}\right)$
}

\begin{abstract}
RESUMO
No Estado de São Paulo, a batata-doce é cultivada em escala comercial como produto hortigranjeiro. Em vista das dificuldades para o controle manual e o mecanizado das plantas infestantes da cultura, há grande interesse pelo método químico desse controle. Com essa finalidade, tanto pelo baixo custo relativo como pelas facilidades técnicas de utilização, o trifluralin desperta a atenção dos agricultores. Para avaliar a seletividade do herbicida para essa cultura, foi realizado um experimento em vasos, com doses crescentes do produto, nas seguintes concentrações: 0,$00 ; 0,11$; 0,$22 ; 0,44 ; 0,88 ; 1,76$ e 3,52 mg.kg-1 de i.a. em peso. Os resultados mostraram que, a partir da dose de $0,22 \mathrm{mg} . \mathrm{kg}^{-1}$ de i.a., o trifluralin interferiu negativamente e em escala exponencial sobre os parâmetros: comprimento das ramas, número de folhas, peso da massa seca da parte aérea e das raizes, avaliados aos 30 dias, revelando-se não seletivo para a batata-doce quando incorporado ao solo, em profundidades semelhantes às da zona de enraizamento das plantas.
\end{abstract}

Termos de indexação: batata-doce, trifluralin, concentração, seletividade.

\section{ABSTRACT \\ SELECTIVITY OF SOIL INCORPORATED TRIFLURALIN FOR SWEET POTATO}

In the State of São Paulo, Brazil, sweet potato is nowadays a commercial vegetable crop. When labor is a limiting factor and the mechanical weed control is difficult, the chemical control is desirable. Trifluralin is an efficient herbicide for weed control in several crops. A green house experiment conducted at the Instituto Agronomico, Campinas, determined the selectivity of trifluralin in sweet potato. Increasing doses of trifluralin $(0.00 ; 0.11 ; 0.22 ; 0.44 ; 0.88 ; 1.76$ and 3.52 $\mathrm{mg.kg} \mathrm{kg}^{-1}$ a.i. in weight) were soil incorporated, uniformly. During thirty days, stem

( ${ }^{\mathrm{l}}$ Trabalho apresentado no XVIII Congresso Brasileiro de Herbicidas e Plantas Daninhas, realizado em Brasília, DF, em junho de 1991. Recebido para publicação em 30 de abril e aceito em 2 de dezembro de 1993.

( ${ }^{2}$ ) Seção de Raizes e Tubérculos, Instituto Agronômico (IAC), Caixa Postal 28, 13001-970 Campinas (SP).

$\left({ }^{3}\right)$ Departamento de Ciências Exatas, FCAV/UNESP, Campus de Jaboticabal, 14870-000 Jaboticabal (SP).

(4) Com bolsa de doutorado da CAPES.

$\left({ }^{5}\right)$ Com bolsa de pesquisa do CNPq. 
cuttings of sweet potatoes were grown in pots. The trial showed that no crop injury occurred by applying $0.11 \mathrm{mg} \cdot \mathrm{kg}^{-1}$ a.i. An exponential injury has been observed at doses upper to $0.22 \mathrm{mg} \cdot \mathrm{kg}^{-1}$ a.i. for the parameters analyzed: stem lenght, leaf number, dry weight of aerial parts and roots. Trifluralin incorporated in soil was a nonselective herbicide for sweet potato plant.

Index terms: sweet potato, trifluralin, concentration, herbicide selectivity.

\section{INTRODUÇÃO}

No Estado de São Paulo, a batata-doce é cultivada comercialmente para o mercado hortigranjeiro. Em valor econômico, situa-se entre os 15 principais entre os 36 legumes mais comercializados nas Centrais de Abastecimento (CEAGESP, 1991). Embora o volume comercializado, no ano, nesses mercados organizados seja de 25.000 toneladas (CEAGESP, 1991), admite-se que, pelo menos outro tanto venha sendo produzido e utilizado pelos próprios produtores ou comercializado marginalmente.

A condição de atividade agrícola comercial passa a exigir capacidade competitiva, onde é fundamental a aplicação prática do conceito da produtividade máxima econômica. $O$ controle da comunidade de plantas infestantes, além de constituir um dos itens de elevada participação no custo total de produção, influi decisivamente na produtividade e na qualidade, quando feito fora dos seus períodos críticos (Unamma et al., 1985).

Devido ao hábito de crescimento da batata-doce, os cultivos mecânicos sem provocar injúrias às plantas só podem ser realizados durante as primeiras semanas após o plantio (Glaze et al., 1981), induzindo à opção pelo controle químico das plantas infestantes nas culturas comerciais.

O trifluralin (2,6-dinitro-N, N-dipropil-4-trifluorometilanilina), por se tratar de um produto de aplicação no solo com incorporação antes do plantio (Blanco et al., 1982), vem despertando grande interesse dos produtores, principalmente pela sua efetividade no controle de plantas infestantes, em especial da família Poaceae. Sua aplicação no solo, porém, antes da confecção dos camalhões para o plantio da batata-doce, pode ser um problema, pois é possivel ocorrer sua acumulação na zona de enraizamento das plantas. Dessa forma, sua seletividade poderá ser reduzida, já que um dos mecanismos de seletividade a esse grupo químico (dinitroanilinas) é o de posição.

Em 1964, Hicks \& Fletchall, citados por Oliver \& Frans (1968), sugeriram que, para a cultura do algodão, a profundidade de incorporação do trifluralin deveria ser levemente inferior à de plantio. Para a mandioca, a incorporação do trifluralin em profundidades semelhantes e até superiores à de plantio não afetou a produtividade da cultura (Doll et al., 1979). Outros autores observaram que a inibição do crescimento das raizes laterais é mais afetada pela profundidade de incorporação do trifluralin do que pela dose de aplicação (Standifer \& Thomas, 1965; Anderson et al., 1967, e Oliver \& Frans, 1968). Essas contradições podem ser explicadas pela existência de outros mecanismos de seletividade para o herbicida. Alguns trabalhos já mostraram haver estreita relação entre os maiores teores de lipídios nos grãos e nas raizes das plântulas e a menor suscetibilidade às dinitroanilinas (Hilton \& Cristiansen, 1972; Ndon \& Harvey, 1981).

Com o propósito de estudar preliminarmente a tolerância da batata-doce a doses crescentes do trifluralin, incorporado de maneira uniforme no perfil do solo, instalou-se um experimento em condições de casa de vegetação.

\section{MATERIAL E MÉTODOS}

O experimento foi desenvolvido em condições de casa de vegetą̧ão, na Seção de Raízes e Tubérculos do Instituto Agronômico em Campinas (SP). Utilizaram-se vasos com $5,5 \mathrm{~kg}$ de terra da camada superficial de um solo hidromórfico, 
com as seguintes características químicas e físicas: pH em água 6,$1 ;$ M.O. $3,6 \% ; \mathrm{P} 215$ mg.kg-1 $\mathrm{Ca}^{+2} 10,1, \mathrm{Mg}^{+2} 2,3, \mathrm{~K}^{+} 0,45 \mathrm{em} \mathrm{meq} / 100 \mathrm{ml}$ de terra fina seca ao ar (TFSA) e argila $35,5 \%$, limo $10,4 \%$, areia fina $25,7 \%$ e areia grossa $28,2 \%$.

O delineamento estatístico utilizado foi de blocos ao acaso, com quatro repetições, sete tratamentos e um vaso por parcela. Os tratamentos constituíram-se da incorporação, no volume total de terra, do trifluralin nas seguintes concentrações: 0,$00 ; 0,11 ; 0,22 ; 0,44 ; 0,88 ; 1,76$ e $3,52 \mathrm{mg} \cdot \mathrm{kg}^{-1}$ em peso.

As ramas utilizadas no plantio foram da parte apical da planta, com cinco folhas expandidas, eliminando-se as quatro primeiras, da base para o ápice.

$O$ trifluralin foi aplicado em $18 / 4 / 89$, com incorporação imediata ao solo. O plantio foi realizado com o auxílio do "plantador-bengala", enterrando-se a parte basal de modo a deixar apenas a folha expandida e o ápice fora da terra.

Aos 30 dias do plantio, efetuou-se a colheita, tendo-se avaliado o comprimento total das ramas (somatório de todas as ramificações emitidas pela planta), o número de folhas expandidas e a massa de matéria seca da parte aérea e do sistema radicular.

\section{RESULTADOS E DISCUSSÃo}

O quadro 1 indica diferenças significativas, ao nível de $1 \%$, para todos os parâmetros analisados (massa seca da parte aérea e do sistema radicular, comprimento de rama e número de folhas), os quais decresceram rapidamente com o aumento da concentração do herbicida.

Se considerarmos uma pulverização ao nivel de campo, utilizando $960 \mathrm{~g}$ de i.a./hectare de trifluralin, recomendada para esse tipo de solo (Almeida \& Rodrigues, 1988), e que, no processo de incorporação associado à confeç̧ão dos cama-

Quadro 1. Efeitos dos incrementos das concentrações de trifluralin incorporado ao solo, sobre plantas de batata-doce cultivadas em vasos. Massa seca da parte aérea, massa seca do sistema radicular, comprimento de rama e número de folhas ( ${ }^{1}$ )

Tratamentos

Massa seca
da parte aérea

Folhas

\begin{tabular}{|c|c|c|c|c|}
\hline $\mathrm{mg} \mathrm{kg}^{-1}$ & & 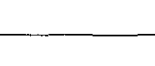 & $\mathbf{m}$ & $\mathbf{n}^{\mathbf{O}}$ \\
\hline 0,00 & $9,26 \mathbf{a b}$ & $2,05 a$ & $1,50 \mathbf{a}$ & $39,5 \mathbf{a}$ \\
\hline 0,11 & $10,00 \mathrm{a}$ & $1,57 \mathrm{ab}$ & $1,63 \mathrm{a}$ & $40,8 \mathrm{a}$ \\
\hline 0,22 & $6,97 \mathrm{bc}$ & $1,22 b$ & $1,37 \mathbf{a}$ & $37,3 \mathbf{a}$ \\
\hline 0,44 & $4,84 \mathrm{~cd}$ & $1,03 \mathrm{bc}$ & $0,78 b$ & $20,3 b$ \\
\hline 0,88 & $3,72 \mathrm{de}$ & $0,52 \mathrm{~cd}$ & $0,46 \mathrm{bc}$ & $13,5 b c$ \\
\hline 1,76 & $2,35 \mathrm{de}$ & $0,18 d$ & $0,15 c$ & $7,0 \mathrm{c}$ \\
\hline 3,52 & $1,78 \mathrm{c}$ & $0,11 d$ & $0,11 \mathrm{c}$ & $5,0 \mathrm{c}$ \\
\hline Tratamento & $34,07^{* *}$ & $24,00 * *$ & $55,00^{* *}$ & $67,52 * *$ \\
\hline D.M.S. (5\%) & 2,58 & 0,69 & 0,39 & 8,76 \\
\hline C.V. $(\%)$ & 20,13 & 31,25 & 20,14 & 16,32 \\
\hline
\end{tabular}

( $\left.{ }^{1}\right)$ Médias de quatro repetições. Quando seguidas por letras iguais não diferem estatisticamente entre si (Tukey, 5\%). ** Significativo ao nível de $1 \%$. 
lhões para o plantio, o herbicida ficasse uniformemente distribuído nos $20 \mathrm{~cm}$ do perfil do solo, a concentração corresponderia a aproximadamente $0,44 \mathrm{mg} \cdot \mathrm{kg}^{-1}$ do produto. Pelos dados do quadro 1 , nota-se que, já nessa concentração, todos os parâmetros analisados foram significativamente afetados pelo herbicida.

Os dados obtidos aos 30 dias após o plantio mostraram que, para os parâmetros analisados, o decréscimo dos valores com o aumento da concentração do herbicida é exponencial, sendo a seguinte equação que se ajusta adequadamente:

$$
\mathrm{Y}=\mathrm{A}+\mathrm{A}_{0} \mathrm{e}^{-\mathrm{kx}} \text {, }
$$

onde:

$\mathrm{Y}$ é o parâmetro analisado; $\mathrm{x}$, a concentração do herbicida, $\mathrm{A}+\mathrm{A}_{0}$, o valor teórico
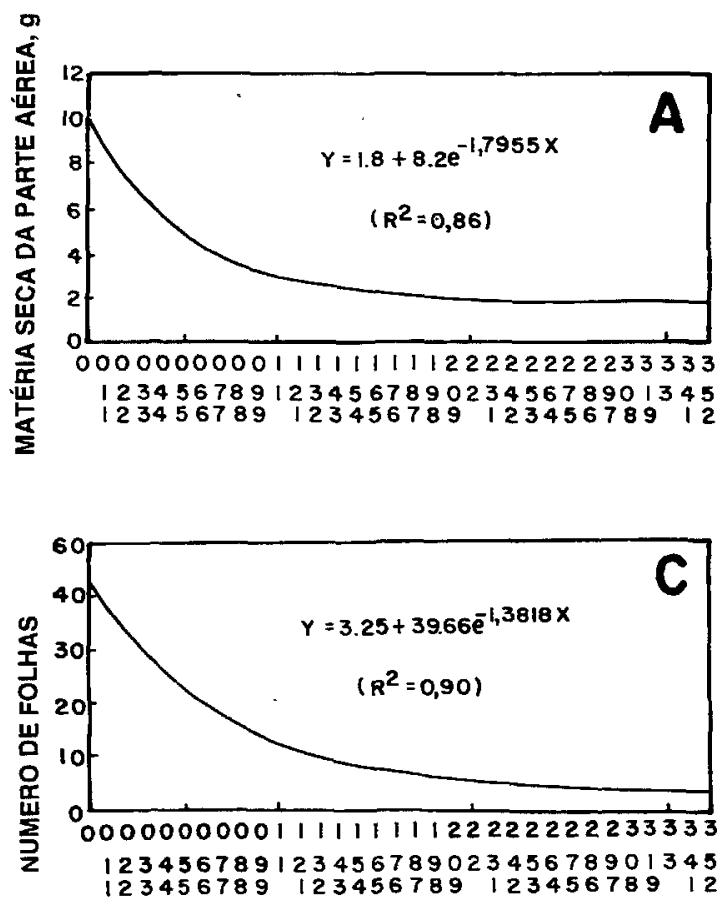

do parâ-metro quando $\mathrm{x}$ tende a zero; $\mathrm{A}$, o valor teórico do parâmetro quando $\mathrm{x}$ tende ao infinito e $k$, a constante de proporcionalidade do decréscimo em função do valor atual.

A figura 1 mostra que a massa do sistema radicular $(k=-2,0000)$ é, entre os parâmetros avaliados, aquele que diminui mais rapidamente com o aumento da concentração do herbicida aplicado, seguido pelo peso de matéria seca da parte aérea $(\mathrm{k}=-1,7955)$, pelo número de folhas $(k=-1,3818)$ e pelo comprimento das ramas $(k=-1,3182)$. Ashton \& Crafts (1973) relatam que os herbicidas do grupo das dinitroanilinas, quando absorvidos pelas raízes, inibem o seu crescimento e o da parte aérea de muitas espécies e que, nesse caso, a inibição do crescimento da parte aérea é provavelmente um efeito secundário, relacionado às limitações do sistema radicular.
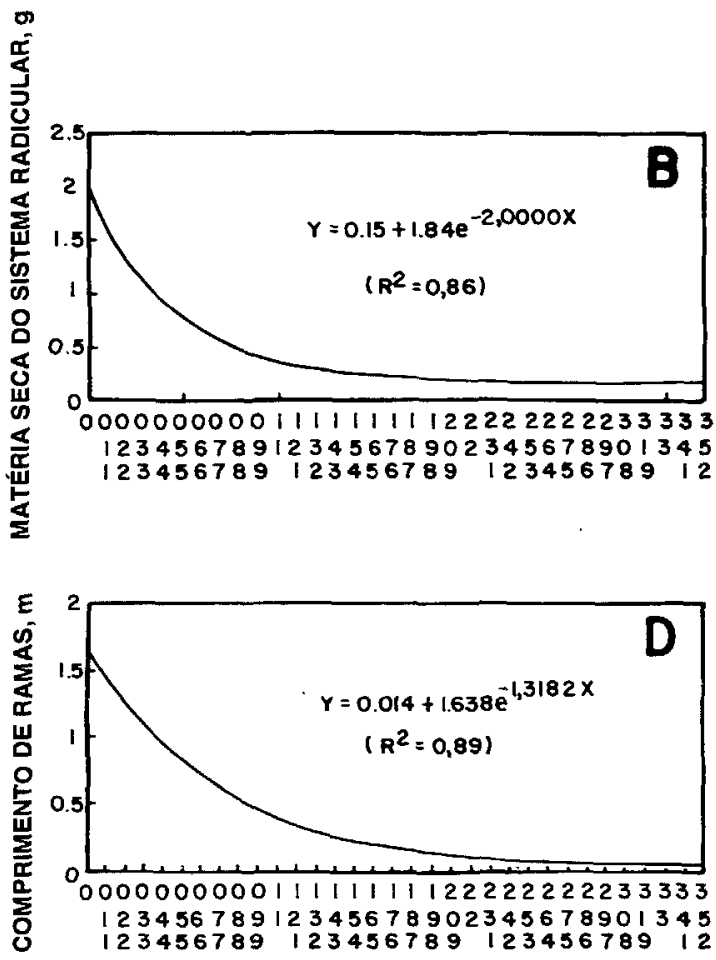

\section{CONCENTRAÇĀO DE TRIFLURALIN, $\mathbf{m g} / \mathrm{kg}^{-1}$}

Figura 1. Efeitos do incremento das concentrações de trifluralin incorporado ao solo sobre a planta de batata-doce, 30 dias após o plantio. 

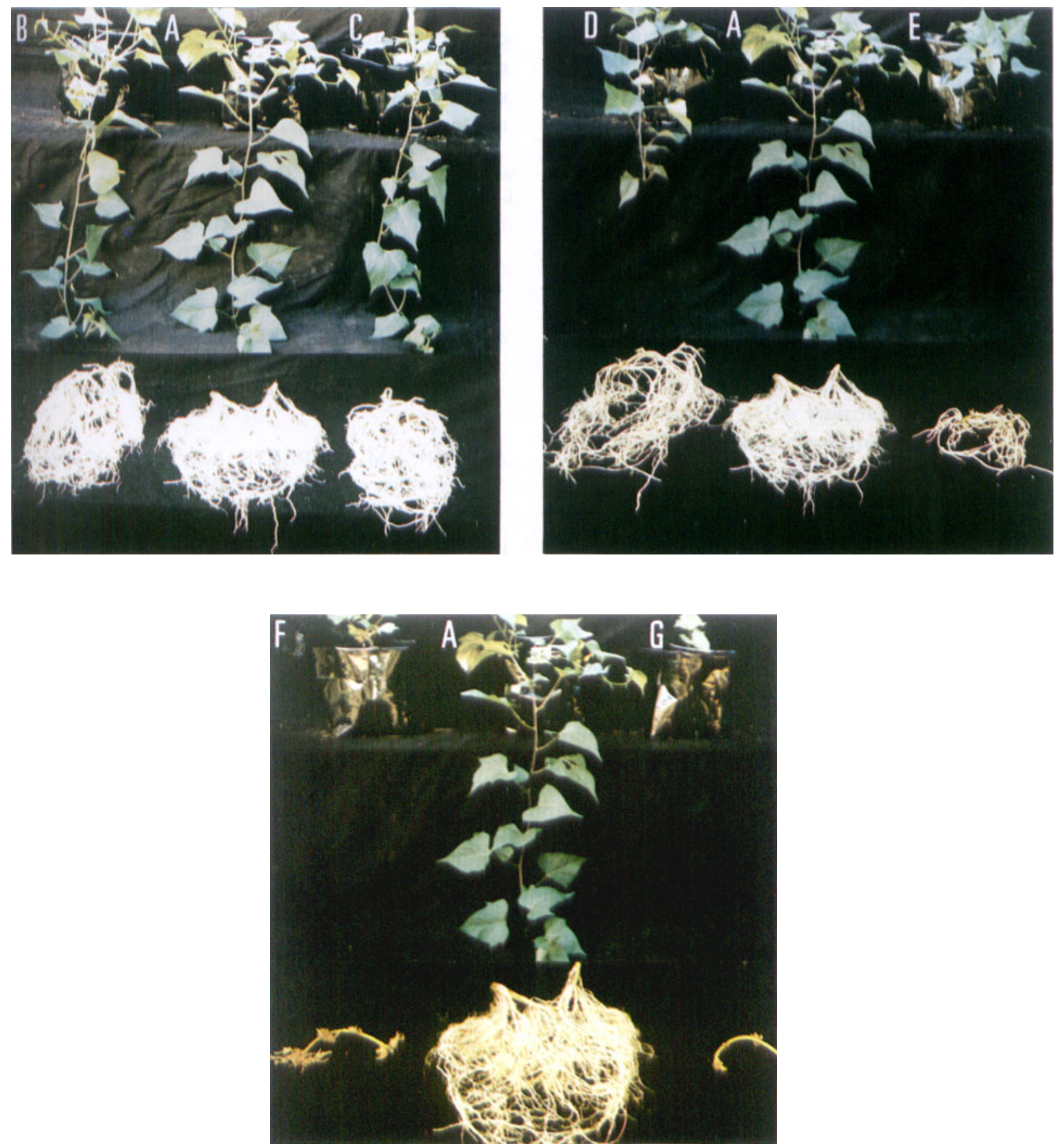

Figura 2. Efeitos lo incremento das concentrações de trifluralin, mg. $\mathrm{kg}^{-1}$, incorporado ao solo sobre o sistema radicular e parte aérea das plantas de batata-doce, 30 dias após o plantio. A: 0,$0 ;$ B: 0,$11 ;$ C: 0,$22 ;$ D: 0,$44 ;$ E: 0,$88 ;$ F: 1,76 e G: 3,52 . 
Parka \& Soper (1977) sugerem que os herbicidas do grupo das dinitroanilinas localizados na zona das raízes são por estas absorvidos ou adsorvidos, sendo minima sua translocação para a parte aérea das plantas. Entretanto, isso é controvertido; a absorção e a translocação das dinitroanilinas não têm sido adequadamente pesquisadas para se chegar a uma conclusão sobre o tema (Ashton \& Crafts, 1973).

\section{Pela equação $\mathrm{Y}=0,15+1,84 \mathrm{e}^{-2,0000 \mathrm{x}}$} (Figura 1B), a concentração de trifluralin para atingir o Iso nas raízes de batata-doce é de $0,39 \mathrm{mg} \cdot \mathrm{kg}^{-1}$. De acordo com Ndon \& Harvey (1981), para a soja, planta considerada resistente, foram necessários $2,62 \mathrm{mg} \cdot \mathrm{kg}^{-1}$ de trifluralin nas raizes para atingir o $\mathrm{I}_{50}$, enquanto, para 0 sorgo, planta considerada suscetível, bastou apcnas $0,17 \mathrm{mg} \cdot \mathrm{kg}^{-1}$, sugerindo ser a batata-doce suscetível a esse herbicida.

Na figura 2 são apresentadas as fotografias referentes ao sistema radicular e à partc aćrca das plantas de batata-doce na colheita do ensaio: em baixas concentrações do herbicida, houve inibição do desenvolvimento das raízes laterais ou secundárias. Segundo Parka \& Soper (1977), essa inibição é uma característica desse grupo de herbicidas, tendo sido relatada por diversos autores em diferentes espécies vegetais.

Bayer et al. (1967) sugeriram que as cćlulas do periciclo e do endoderma são mais afetadas pelo trifluralin do que as do meristema apical da raiz. Segundo Durigan et al. (1986/91), essa inibição do crescimento das raízes secundárias e do desenvolvimento da parte aérea das plantas é a modificação morfológica mais freqüentc que ocorre em função do aumento das doses, profundidades de incorporação e outros fatores que possam levar à perda de seletividade. Aumentando a concentração de trifluralin no solo, observou-se, na batata-doce, desde a inibição das raízes laterais ou secundárias até o ponto em que estas não mais se desenvolveram, acompanhado de um aumento do diâmetro das raízes adventícias principais. (Figura 2).

\section{CONCLUSÃo}

O trifluralin quando incorporado ao solo, em profundidades semelhantes às da zona de enraizamento da cultura, nas concentrações de utilização do herbicida em condições de campo, não apresentou seletividade para a batata-doce.

\section{REFERÊNCIAS BIBLIOGRÁFICAS}

ALMEIDA, F.S. \& RODRIGUES, B.N. Guia de herbicidas. 2.ed. Londrina, 1988. 603p.

ANDERSON, W.P.; RICHARDS, A.B. \& WHITWORTH, J.W. Trifluralin effects on cotton seedlings. Weed Science, Champaign, 15(3):224-227, 1967

ASIITON, F.M. \& CRAFTS, A.S. Mode of action of herbicides. New York, Wiley Interscience, 1973. $504 \mathrm{p}$.

BAYER, D.E.; FOY, C.L.; MAIIROY, T.E. \& CUTTER, I.G. Morphological and histological effects of trifluralin on root development. American Journal of Botany, New York, 54(8):945-952, 1967.

BL.ANCO, H.G.; NOVO, M.C.S.S.; COELHO, R.R. \& OLIVEIRA, D.A. Comportamento do herbicida trifluralina em solos. III. Persistência em solos arcnosos cultivados com soja, na região de Pirassununga, Estado de São Paulo. O Biológico, São Paulo, 48(9):217-221, 1982.

BOLETIM ANUAL CEAGESP. São Paulo, 1991.

DOLL, J.D.; PIEDRAHÍTA, W.C. \& LEIHNER, D.E. Métodos de control de malezas en yuca (Manihot esculenta Crantz). In: DOMÍNGUEZ, C.E., coord. Yuca: investigación, producción y utilización. Cali, Programa de las Naciones Unidas para el Desarrollo/Centro Internacional de Agricultura Tropical, 1979. cap.3, p.241-249.

DURIGAN, J.C.; MATUO, T.; ANDRADE, V.M.R.M. \& FERREIRA, J.C. Reação de plantas de amendoim (Arachis hypogaea L.) ao trifluralin e mudanças na morfologia da parte subterrânea. Planta Daninha, Campinas, 9(1/2):12-26, 1986/91.

GLAZE, N.C.; HARMON, S.A. \& PLATAK, S.C. Enhancement of herbicidal weed control in sweet potatoes (Ipomoea batatas) with cultivation. Weed Science, Champaign, 29(3):275-281, 1981. 
HILTON, J.L. \& CHRISTIANSEN, M.N. Lipid contribution to seletive action of trifluralin. Weed Science, Champaign, 20(4):290-294, 1972.

NDON, B.A. \& HARVEY, R.G. Effects of seed and root lipids on the susceptibility of plants to trifluralin and oryzalin. Weed Science, Champaign, 29(3):420-425, 1981.

OLIVER, L.R. \& FRANS, R.E. Inhibition of cotton and soybean roots from incorporated trifluralin and persistence in soil. Weed Science, Champaign, 16(2): 199-203, 1968.
PARKA, S.J. \& SOPER, O.F. The Physiology and mode of action of the dinitroaniline herbicides. Weed Science, Champaign, 25(1):79-87, 1977.

STANDIFER JUNIOR, L.C. \& THOMAS, C.H. Response of johnsongrass to soil incorporated trifluralin. Weeds, Urbana, 13(4):302-306, 1965.

UNAMMA, R.P.A.; ENYINNIA, T. \& EMEZIE, S.F. Critical period of weed interference in cocoyan/ /maize/sweet potato intercrop. Tropical Pest Management, London, 31(1):21-23, 1985. 